\title{
Replacing A Lateral Incisor Using SMART Base
}

Massa $\mathrm{L}^{1 *}$ and Fraunhofer $\mathrm{JAV}^{2}$

${ }^{1}$ Premier Dental Centers, USA

${ }^{2}$ School of Dentistry, University of Maryland, USA

*Corresponding author: Massa L, Premier Dental Centers, USA

\section{Editorial}

Replacing a missing lateral incisor always presents a dentist with various challenges, notably the need to ensure an esthetic result and long-term clinical viability in restoring oral health and functionality. Due to concavities in the premaxilla region the implant apex is often placed with a cant to the palate. This dilemma can be solved with custom abutments which increase the cost of the case. The SMART base allows up to 25 degrees of angle correction which allows for screw retention in these cases (Figure 1). Figure 2 shows a radiograph of a missing lateral incisor and a pre-operative template of a wax-up. An implant is then placed in the jaw and allowed to heal for 6-8 weeks to ensure osseointegration (Figure
3). Thereafter, a temporary abutment is placed, adjusted and after the screw is removed, a cotton pellet is placed to close the aperture and protect the abutment threads (Figure 4). The temporary abutment is then "picked-up" using a flowable composite while an emergence profile and screw access is created with the composite resin (Figure 5). The temporary restoration is then placed, and the retaining screw torqued to $15 \mathrm{Ncm}$ (Figure 6). Thereafter, the screw access is closed with a cotton pellet and composite resin (Figure 7). The SMART base can then be scanned (Figures 8) and digitized. The final screw-retained zirconia restoration can then be fabricated using CAD/CAM technology and placed in the mouth (Figure 9). The final result is shown in Figure 10.

Figure 1: Angle correction of the smart base. 


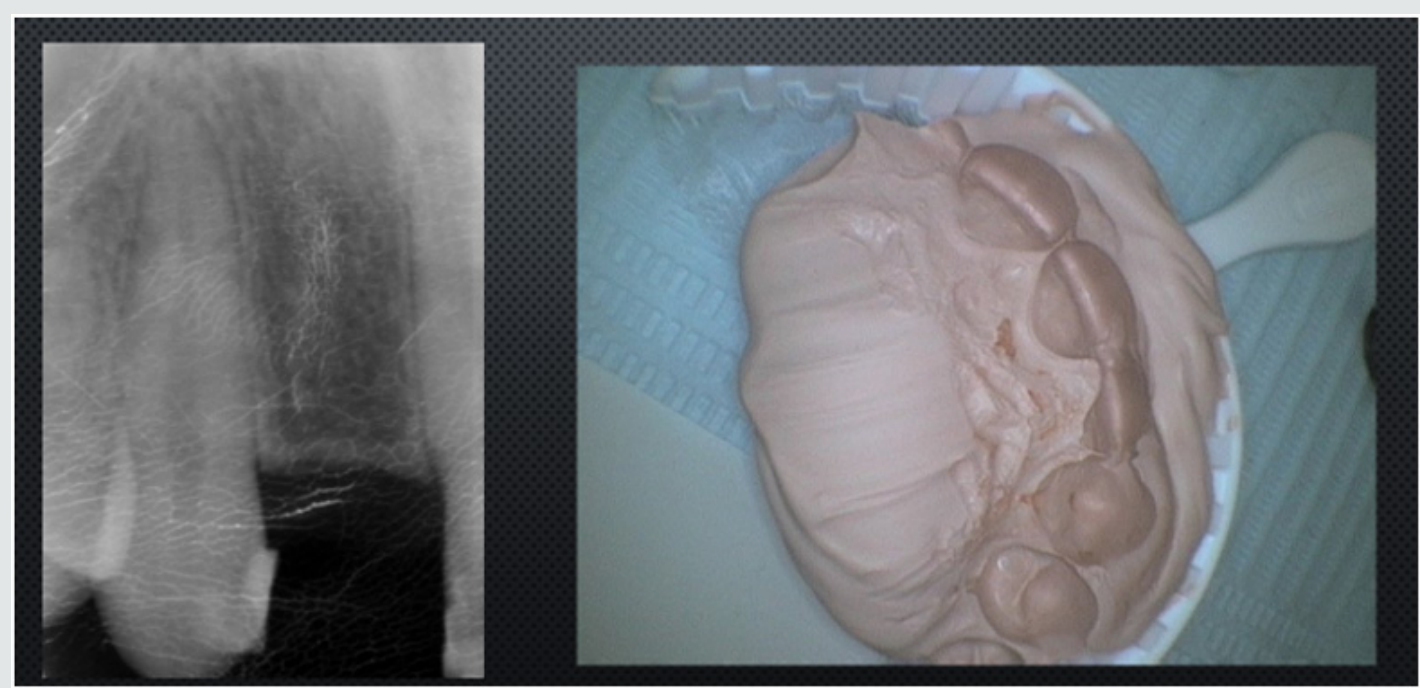

Figure 2: Radiograph and pre-operative template of wax-up.

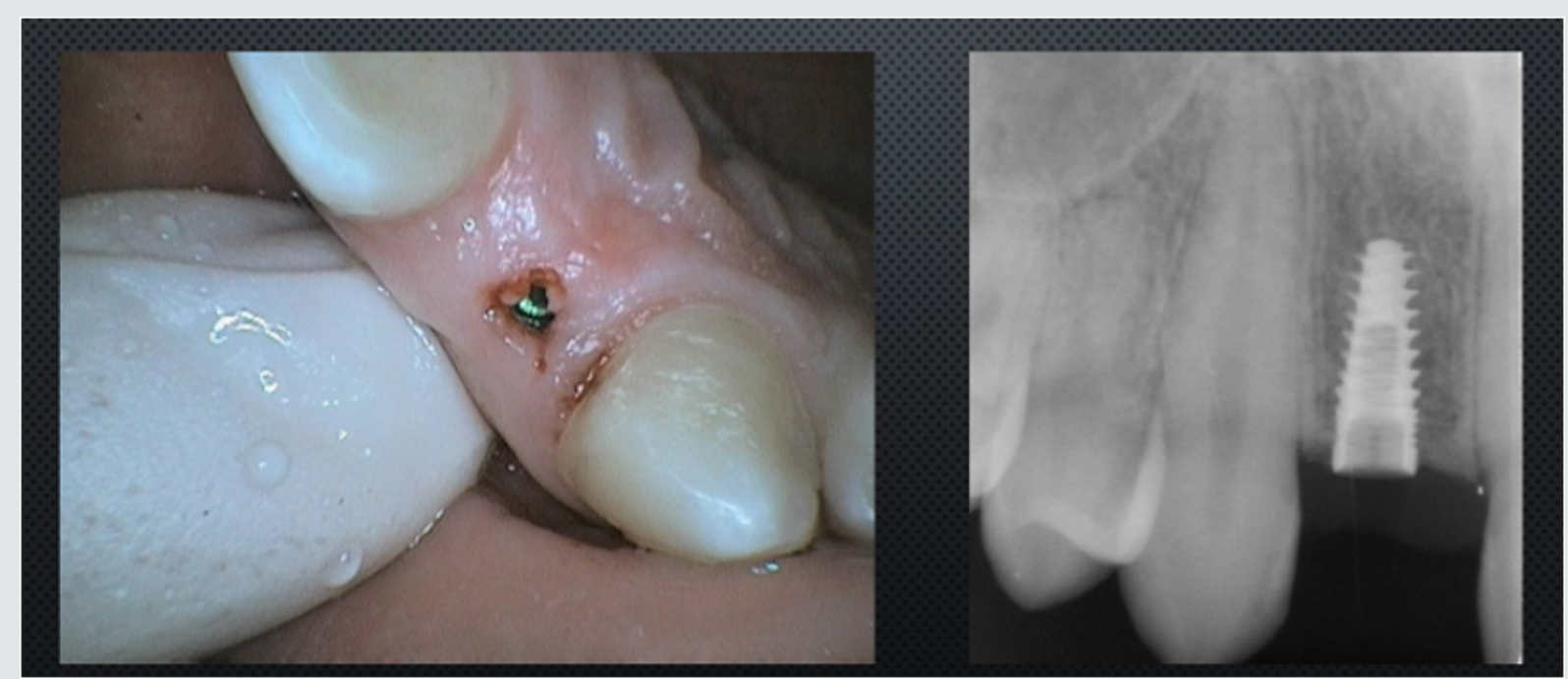

Figure 3: Implant placed and allowed to osseointegrate.

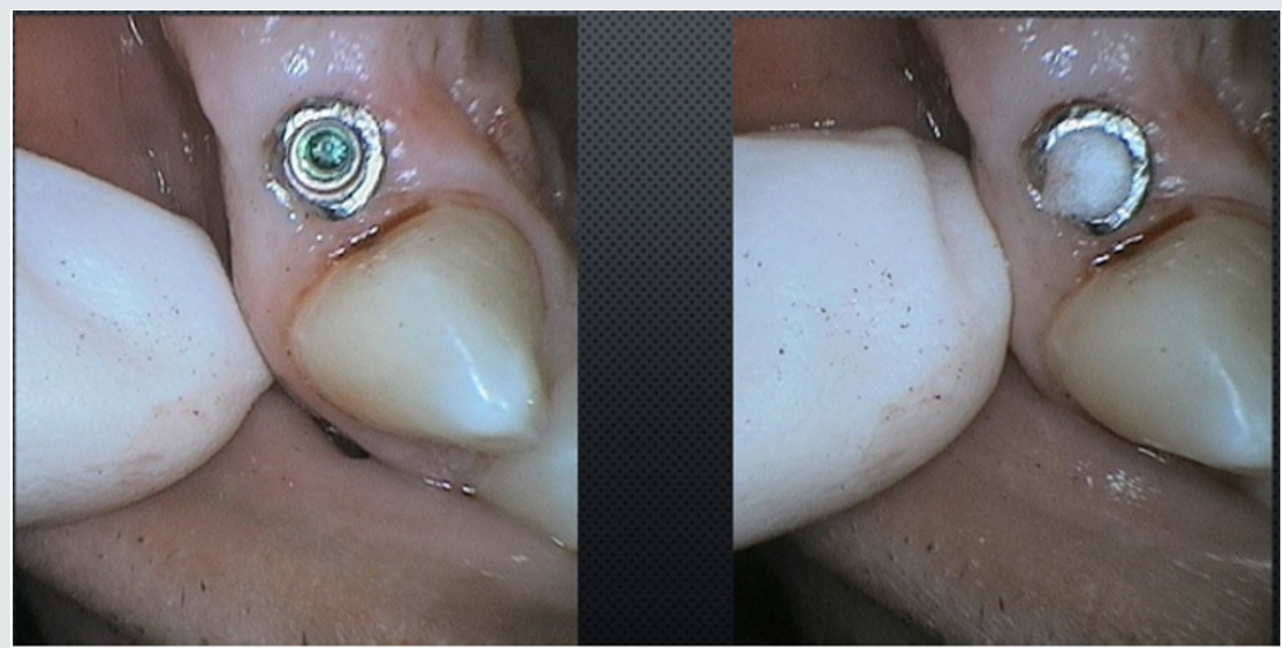

Figure 4: Placement of the temporary abutment, adjustment and protecting abutment aperture and threads with a cotton pellet. 


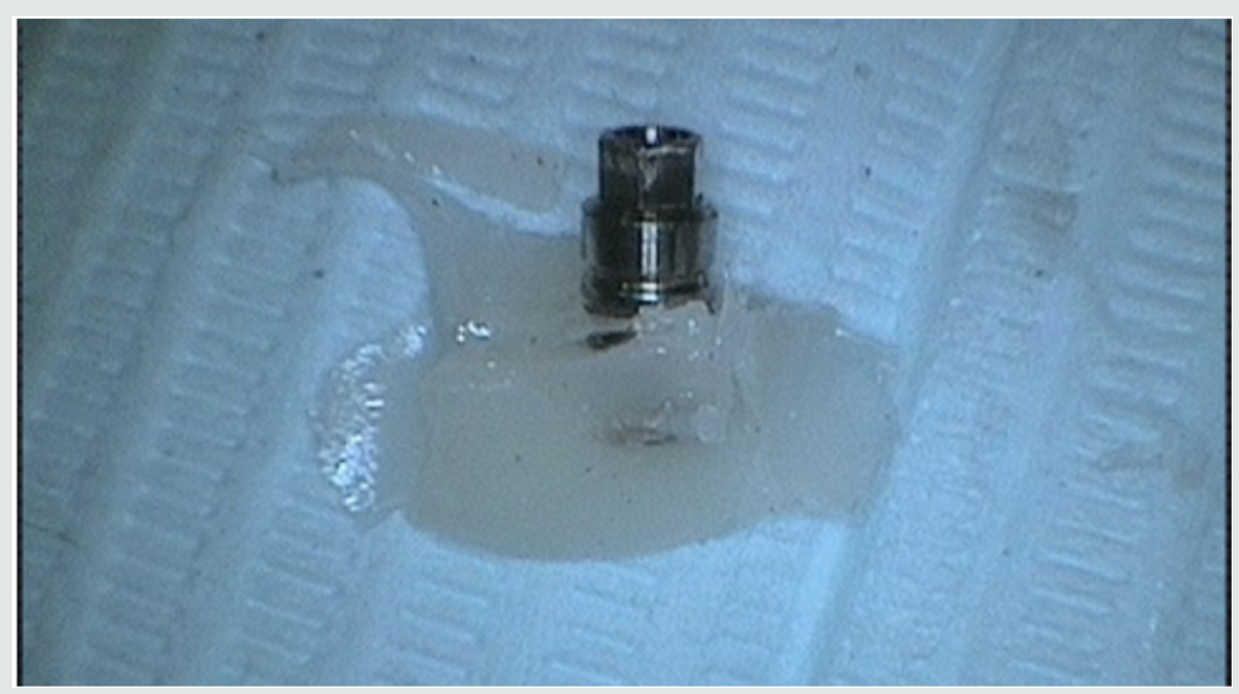

Figure 5: Picking-up the temporary abutment and creating both an emergence profile and screw access.

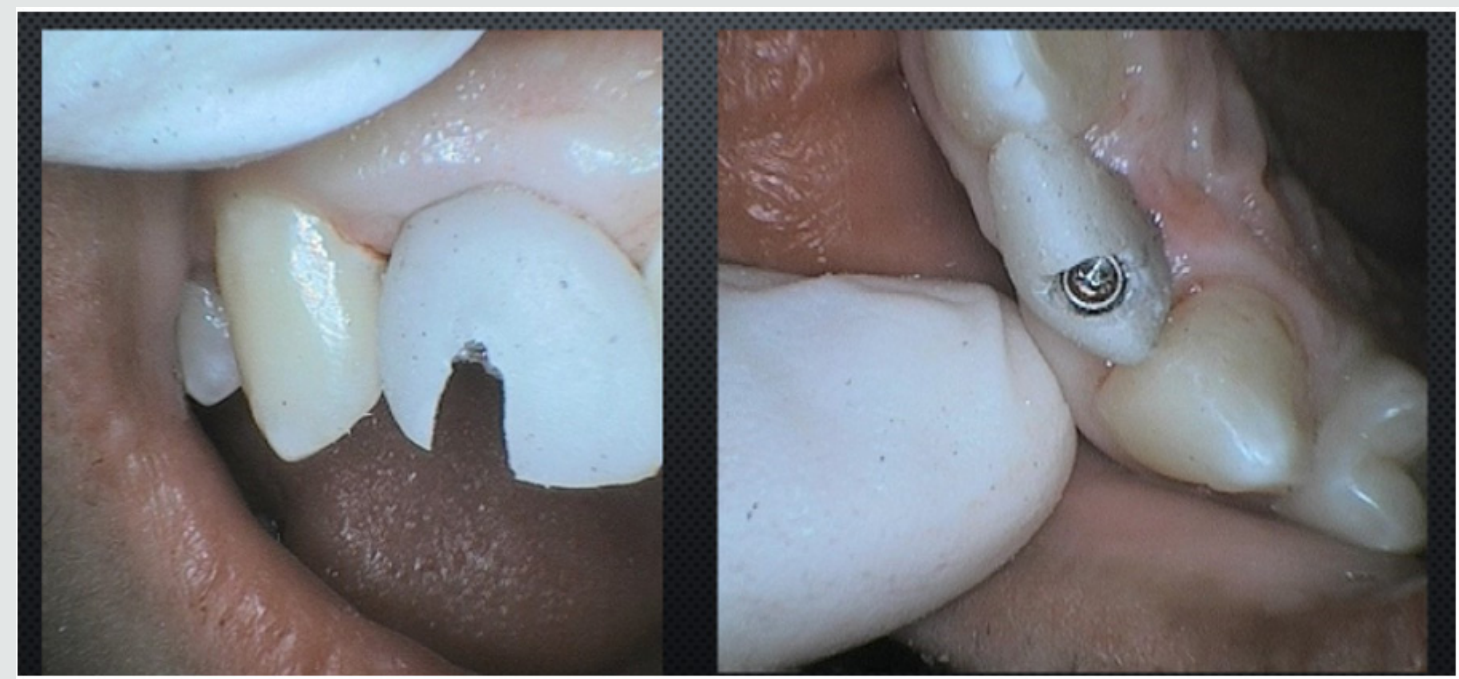

Figure 6: Temporary restoration placed, and the retaining screw torqued to $15 \mathrm{Ncm}$.

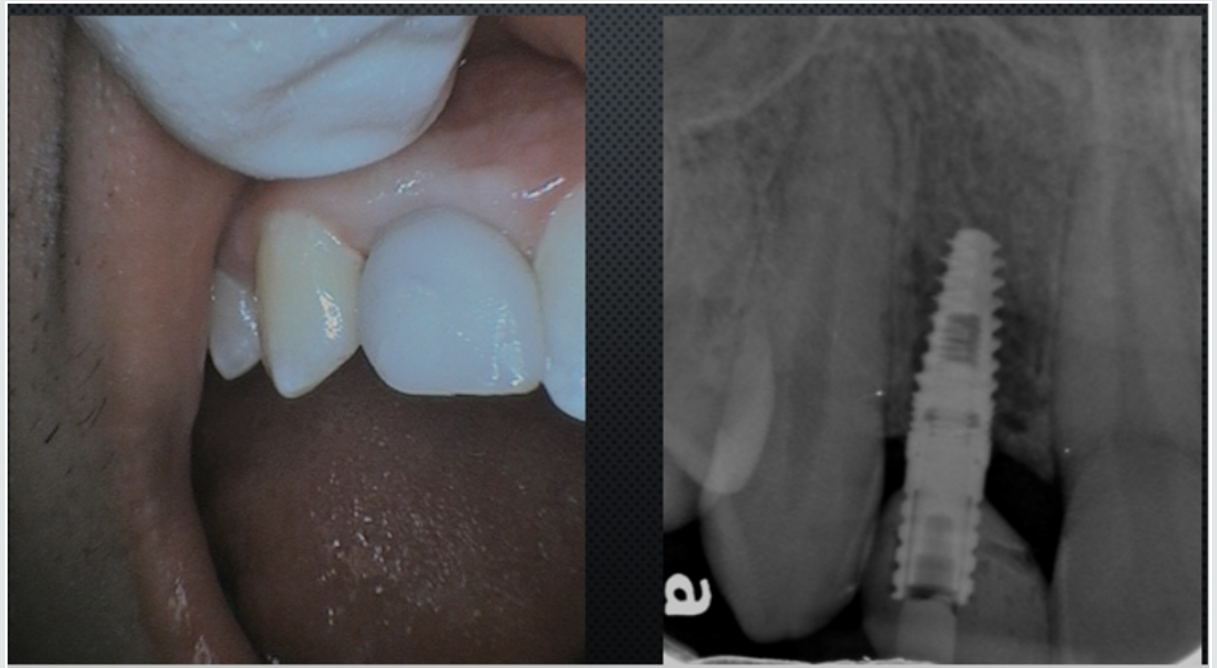

Figure 7: The screw access is closed with a cotton pellet and composite resin. 

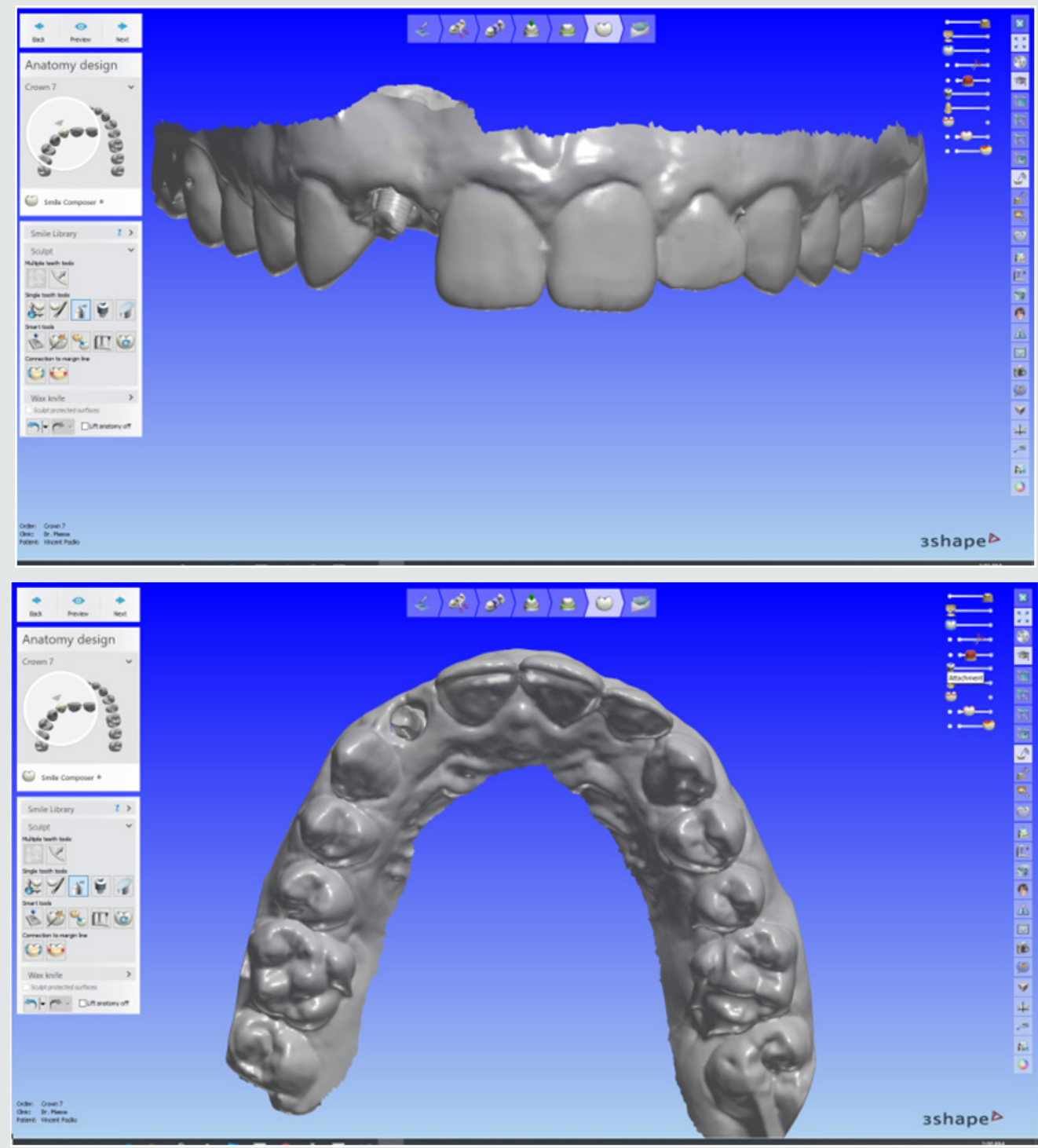

Figure 8: $a$, b Scanned and digitized images of the SMART base.

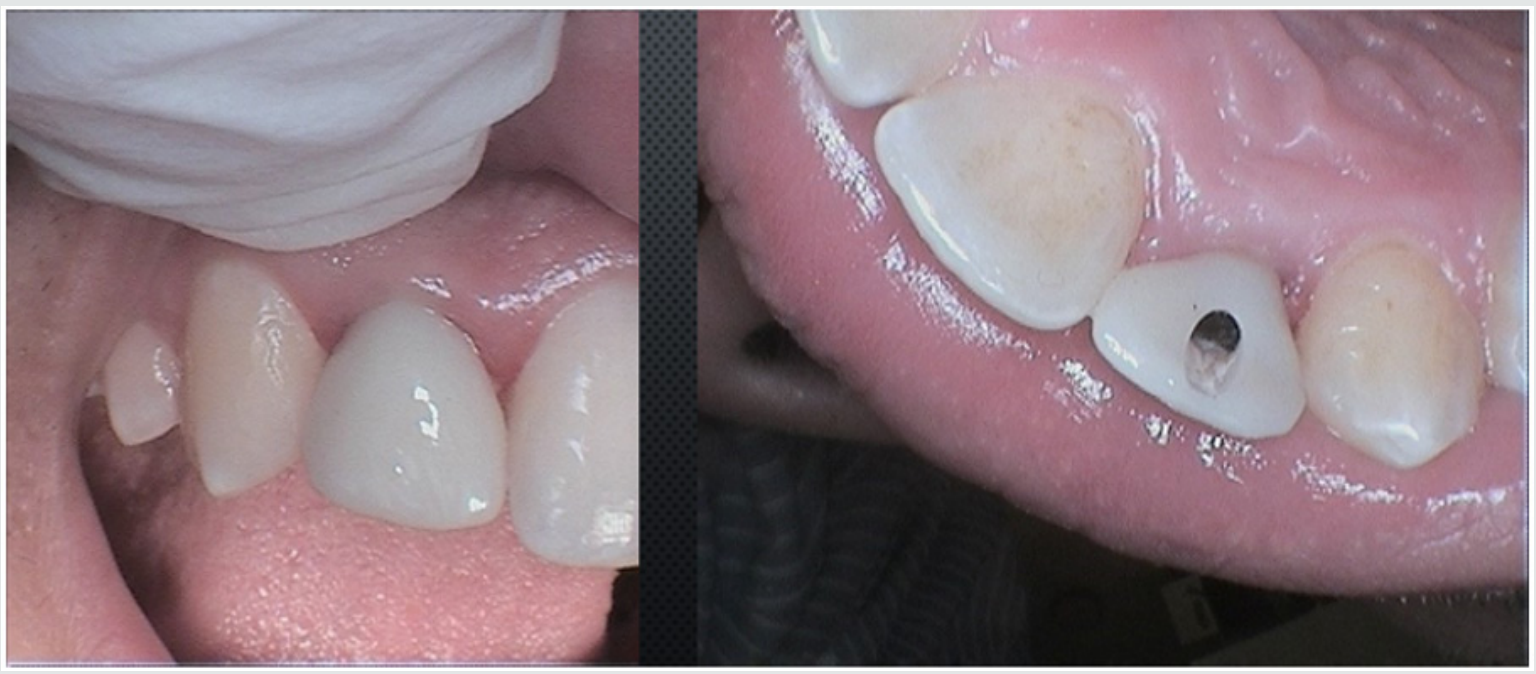

Figure 9: Placement of the final screw-retained zirconia restoration. 


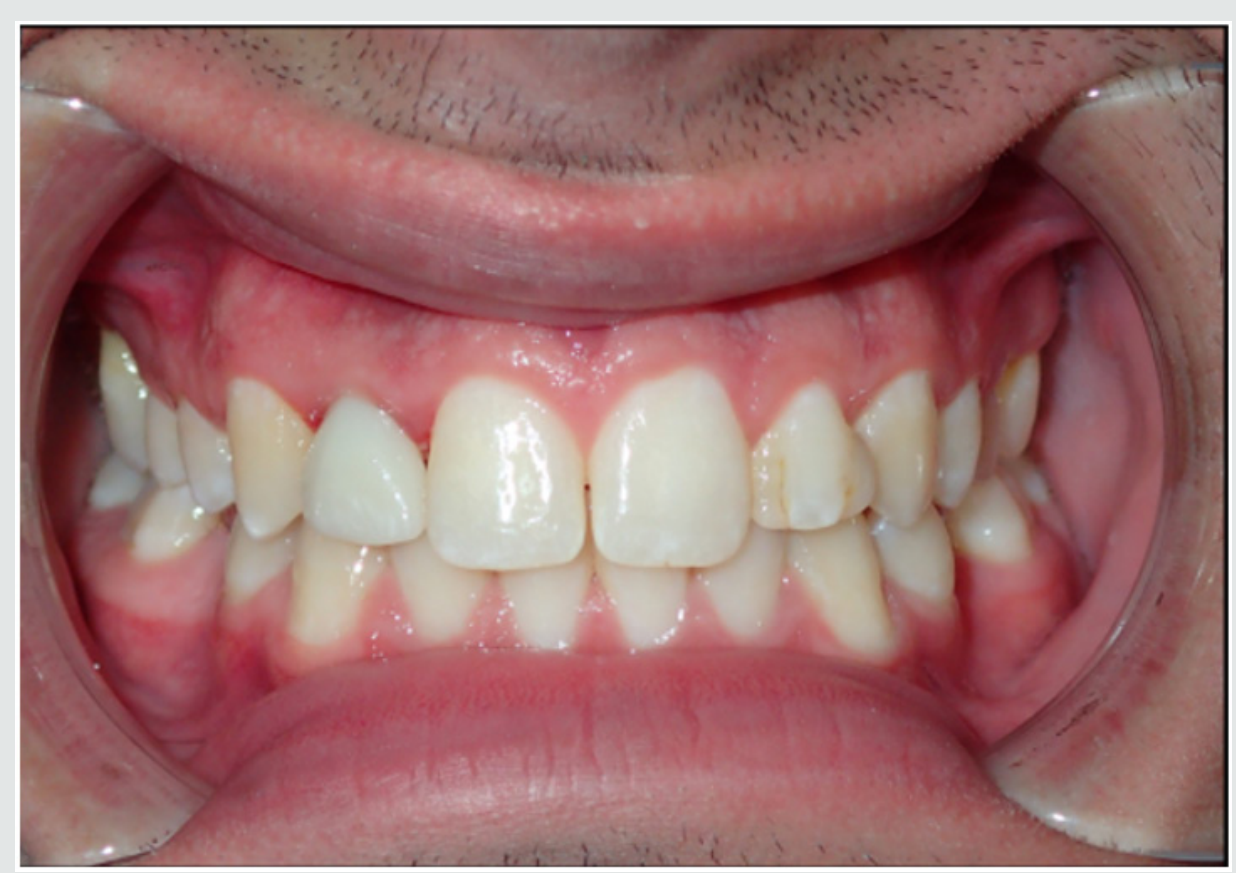

Figure 10: Final result.

The technique outlined here is a simple and direct approach to dealing with a missing lateral incisor and an implant which requires angle correction. The advantage of this methodology is that intraoral procedures are held to minimum whereas maximum advantage is taken of modern dental technology, notably prefabricated abutments, digital scanning and CAD/CAM fabrication of screw- retained restorations. Because modern technology is involved in every step of the treatment planning and restoration fabrication, clinician time is saved, patient discomfort (and expense) is held to a minimum and the restoration will achieve the desired objectives with minimal time wastage.

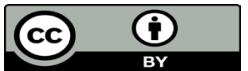

This work is licensed under Creative Commons Attribution 4.0 License

To Submit Your Article Click Here:

Submit Article

DOI: 10.32474/MADOHC.2020.04.000188

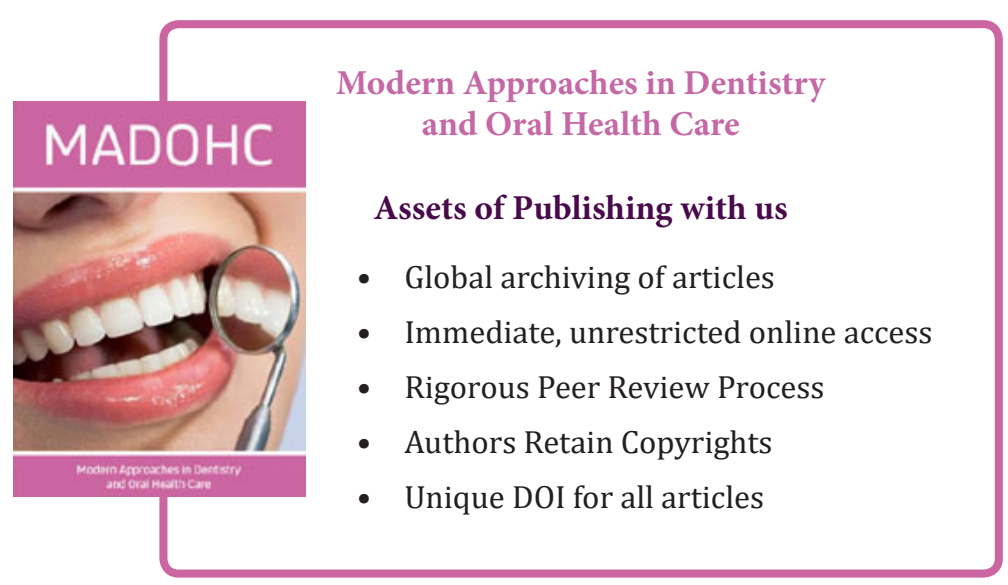

